\title{
Erratum to: Scalarization of constraints system in some vector optimization problems and applications
}

\author{
Marius Durea · Radu Strugariu
}

Published online: 6 July 2014

(C) Springer-Verlag Berlin Heidelberg 2014

\section{Erratum to: Optim Lett \\ DOI 10.1007/s11590-013-0690-x}

We have observed that an assumption was overlooked by us in the statements of Theorems 3.1, 3.3, and 4.3 of the above mentioned paper. We give below the complete formulations of these results. The proofs given in the initial article are now valid.

Theorem 3.1 Suppose that $g: X \rightarrow Z$ is continuous, $Q$ is a closed convex proper and pointed cone, $\bar{x} \in M \cap \operatorname{dom} s$ is a local solution of $\left(P_{s}\right)$, where $s: X \rightarrow R \cup\{+\infty\}$ is locally Lipschitz around $\bar{x}$ relative to its domain with constant $L>0$. If there exists a neighborhood $U$ of $\bar{x}$ such that $U \cap M \subset \operatorname{dom} s$ and $g$ is metrically regular at $\bar{x} \in M=g^{-1}(-Q)$ relative to $-Q$ (with regularity modulus $m>0$ ), then for every $\bar{q} \in Q \backslash\{0\}, \bar{x}$ is a local minimum (without constraints) of the function $s+\operatorname{Lm}\|\bar{q}\| \cdot\left(\mu_{Q, \bar{q}} \circ g\right)$.

Theorem 3.3 Suppose that $\bar{x} \in M$ is a local solution of $\left(P_{v}\right), g: X \rightarrow Z$ is continuous, $v$ is locally Lipschitz around $\bar{x}$ and $\varphi_{K, \bar{k}}$ is Lipschitz relative to its domain. If there exists a neighborhood $U$ of $\bar{x}$ such that $U \cap M \subset v^{-1}(v(\bar{x})+R \bar{k}-K)$

The online version of the original article can be found under doi:10.1007/s11590-013-0690-x.

M. Durea $(\bowtie)$

Faculty of Mathematics, Al. I. Cuza University, Bd. Carol I, nr. 11,

700506 Iasi, Romania

e-mail: durea@uaic.ro

R. Strugariu

Department of Mathematics and Informatics, Gh. Asachi Technical University,

Bd. Carol I, nr. 11, 700506 Iasi, Romania

e-mail: rstrugariu@tuiasi.ro 
and $g$ is metrically regular at $\bar{x}$ relative to $-Q$, then for every $\bar{q} \in Q \backslash\{0\}$, there exists $\eta>0$ s.t. $\bar{x}$ is a local minimum (without constraints) of the function $\varphi_{K, \bar{k}} \circ(v(\cdot)-v(\bar{x}))+\eta\left(\mu_{Q, \bar{q}} \circ g\right)$.

Theorem 4.3 Take $\bar{x} \in M$ as a local solution of $\left(P_{v}\right)$ and take $\bar{k} \in K \backslash\{0\}, \bar{q} \in Q \backslash\{0\}$. Suppose that there exists a neighborhood $U$ of $\bar{x}$ such that $U \cap M \subset v^{-1}(v(\bar{x})+R \bar{k}-$ $K)$ and

(i) $g$ is continuous and metrically regular at $\bar{x}$ relative to $-Q$.

(ii) $v$ is locally Lipschitz around $\bar{x}$ and $\varphi_{K, \bar{k}}$ is Lipschitz relative to its domain.

(iii) the function $h:(X \times R)^{2} \rightarrow X, h(\alpha, \beta, \gamma, \delta):=\alpha-\gamma$ is metrically subregular at the point $\left(\bar{x}, 0, \bar{x}, \mu_{Q, q}(g(\bar{x}))\right)$ with respect to $\operatorname{Gr}\left(\varphi_{K, \bar{k}} \circ(v(\cdot)-v(\bar{x}))\right)_{\uparrow} \times$ $\operatorname{Gr}\left(\mu_{Q, \bar{q}} \circ g\right) \uparrow$.

(iv) $\left(\varphi_{K, \bar{k}} \circ(v(\cdot)-v(\bar{x}))\right)_{\uparrow}$ is proto-differentiable at $\bar{x}$ relative to 0 or $\left(\mu_{Q, \bar{q}} \circ g\right)_{\uparrow}$ is proto-differentiable at $\bar{x}$ relative to $\mu_{Q, q}(g(\bar{x}))$.

Then there exists $\eta>0$ s.t., for any $u \in X$,

$$
D_{\uparrow}\left(\varphi_{K, \bar{k}} \circ(v(\cdot)-v(\bar{x}))\right)(\bar{x})(u)+\eta D_{\uparrow}\left(\mu_{Q, \bar{q}} \circ g\right)(\bar{x})(u) \geq 0 .
$$

